\title{
Some Implications of the Cosmological Constant to Fundamental Physics
}

\author{
R. Aldrovandi*, J P Beltrán Almeida* and J G Pereira* \\ *Instituto de Física Teórica, Universidade Estadual Paulista \\ Rua Pamplona 145, 01405-900 São Paulo, Brazil
}

\begin{abstract}
In the presence of a cosmological constant, ordinary Poincaré special relativity is no longer valid and must be replaced by a de Sitter special relativity, in which Minkowski space is replaced by a de Sitter spacetime. In consequence, the ordinary notions of energy and momentum change, and will satisfy a different kinematic relation. Such a theory is a different kind of a doubly special relativity. Since the only difference between the Poincare and the de Sitter groups is the replacement of translations by certain linear combinations of translations and proper conformal transformations, the net result of this change is ultimately the breakdown of ordinary translational invariance. From the experimental point of view, therefore, a de Sitter special relativity might be probed by looking for possible violations of translational invariance. If we assume the existence of a connection between the energy scale of an experiment and the local value of the cosmological constant, there would be changes in the kinematics of massive particles which could hopefully be detected in high-energy experiments. Furthermore, due to the presence of a horizon, the usual causal structure of spacetime would be significantly modified at the Planck scale.
\end{abstract}

Keywords: de Sitter special relativity, de Sitter spaces, cosmological constant

PACS: $04.20 . \mathrm{Cv} ; 04.50 .+\mathrm{h}$

\section{INTRODUCTION}

When the cosmological constant $\Lambda$ vanishes, absence of gravitation is represented by Minkowski spacetime, a solution of the sourceless Einstein's equation. Its isometry transformations are those of the Poincare group, which is the group governing the kinematics of special relativity. For a non-vanishing $\Lambda$, however, Minkowski is no longer a solution of the corresponding Einstein's equation and becomes, in this sense, physically meaningless. In this case, absence of gravitation turns out to be represented by the de Sitter spacetime. Now, the group governing the kinematics in a de Sitter spacetime is not the Poincaré, but the de Sitter group. This means essentially that, in the presence of a nonvanishing $\Lambda$, ordinary Poincaré special relativity will no longer be valid, and must be replaced by a de Sitter special relativity $[1,2]$. Since the local symmetry is also represented by the de Sitter group, the tangent space at each point of a Riemannian spacetime must also be replaced by an osculating de Sitter space.

An important point of this construction is that it retains the quotient character of spacetime and, consequently, a notion of homogeneity. As in ordinary special relativity, therefore, whose underlying Minkowski spacetime is the quotient space of the Poincare by the Lorentz groups, the underlying spacetime of the de Sitter relativity will be the quotient space of the de Sitter and the Lorentz groups. Now, a space is said to be transitive under a set of transformations - or homogeneous under them - when any two points of it can be attained from each other by a transformation belonging to the set. For example, the Minkowski spacetime is transitive under spacetime translations. However, the de Sitter spacetime is transitive, not under translations, but under a combination of translations and proper conformal transformations, with the relative importance of these contributions being determined by the value of the cosmological constant. Observe that, due to the quotient character of spacetime, it will respond concomitantly to any deformation occurring in the symmetry groups. For small values of $\Lambda$, for example, the de Sitter group will approach the Poincare group, and the underlying spacetime will approach the flat Minkowski spacetime. For large values of $\Lambda$, the de Sitter group will approach the conformal Poincare group, and the underlying spacetime will approach a new maximally-symmetric conic spacetime, which is homogeneous under proper conformal transformations [3].

An immediate consequence of the de Sitter homogeneity properties is that the ordinary notions of energy and momentum will change [4]. In fact, the conserved momentum, for example, will now be obtained from the invariance of the physical system, not under translations, but under a combination of translations and proper conformal transformations. The conserved momentum, therefore, will be given by a combination of ordinary and proper conformal momenta, the relative importance of these contributions being again determined by the value of the cosmological con-

CP910, Cosmology and Gravitation, edited by M. Novello and S. E. Perez Bergliaffa

(C) 2007 American Institute of Physics 978-0-7354-0418-2/07/\$23.00 
stant. Of course, the usual special relativistic relation between ordinary mass, energy and momentum will also change. Another important consequence of the presence of a cosmological constant is that it modifies the usual Lorentz causal structure of spacetime, defined by the light cone. In fact, the causal domain of all observers will be further restricted by the presence of the de Sitter horizon.

To get some insight on how the de Sitter special relativity may be thought of, let us briefly recall the relationship between the de Sitter and the Galilei groups, which comes from the Wigner-Inönü processes of group contraction and expansion $[5,6]$. Ordinary Poincaré special relativity can be viewed as describing the implications to Galilei's relativity of introducing a fundamental velocity-scale into the Galilei group. Conversely, the latter can be obtained from the special-relativistic Poincaré group by taking the formal limit of the velocity scale going to infinity (nonrelativistic limit). We can, in an analogous way, say that de Sitter relativity describes the implications to Galilei's relativity of introducing both a velocity and a length scales in the Galilei group. In the formal limit of the length-scale going to infinity, the de Sitter groups contract to the Poincare group, in which only the velocity scale is present. It is interesting to observe that the order of the group expansions (or contractions) is not important. If we introduce in the Galilei group a fundamental length parameter, we end up with the Newton-Hooke group [7], which describes a (non-relativistic) relativity in the presence of a cosmological constant [8]. Adding to this group a fundamental velocity scale, we end up again with the de Sitter group, whose underlying relativity involves both a velocity and a length scales. Conversely, the low-velocity limit of the de Sitter group yields the Newton-Hooke group, which contracts to the Galilei group in the limit of a vanishing cosmological constant.

The purpose of this article is to discuss the main features and consequences of a de Sitter special relativity. We will proceed as follows. Section 2 is a review of the fundamental properties of the de Sitter groups and spaces. In section 3 it is shown how the transitivity properties of the de Sitter spacetime can lead to two different notions of distance: a translational and a conformal distance. In section 4, the fundamentals of a de Sitter special relativity are presented and discussed. In particular, an analysis of the deformed generators acting on the de Sitter space is made, which allows us to understand how a de Sitter relativity keeps a well defined algebraic structure on a de Sitter spacetime. The modified notions of energy and momentum, as well as the new relationship between them, are obtained in section 5. Finally, section 6 discusses the results obtained. In particular, the relation of the de Sitter relativity with the so called doubly special relativity is discussed, and some speculations on possible phenomenological consequences are examined. Closing the article, an analysis of the new spacetime causal structure at the Planck length is performed.

\section{DE SITTER SPACETIMES AND GROUPS}

Spacetimes with constant scalar curvature $R$ are maximally symmetric: they can lodge the highest possible number of Killing vectors. Given a metric signature, this spacetime is unique [9] for each value of $R$. Minkowski spacetime $M$, with $R=0$, is the simplest one. Its group of motions is the Poincaré group $\mathscr{P}=\mathscr{L} \oslash \mathscr{T}$, the semi-direct product of the Lorentz $\mathscr{L}=S O(3,1)$ and the translation group $\mathscr{T}$. The latter acts transitively on $M$ and its group manifold can be identified with $M$. Indeed, Minkowski spacetime is a homogeneous space under $\mathscr{P}$, actually the quotient

$$
M=\mathscr{P} / \mathscr{L} \text {. }
$$

Amongst curved spacetimes, the de Sitter and anti-de Sitter spaces are the only possibilities [10]. One of them has negative, and the other has positive scalar curvature. They are hyper-surfaces in the "host" pseudo-Euclidean spaces $\mathbf{E}^{4,1}$ and $\mathbf{E}^{3,2}$, inclusions whose points in Cartesian coordinates $\left(\chi^{A}\right)=\left(\chi^{0}, \chi^{1}, \chi^{2}, \chi^{3}, \chi^{4}\right)$ satisfy respectively

$$
\eta_{A B} \chi^{A} \chi^{B} \equiv\left(\chi^{0}\right)^{2}-\left(\chi^{1}\right)^{2}-\left(\chi^{2}\right)^{2}-\left(\chi^{3}\right)^{2}-\left(\chi^{4}\right)^{2}=-l^{2}
$$

and

$$
\eta_{A B} \chi^{A} \chi^{B} \equiv\left(\chi^{0}\right)^{2}-\left(\chi^{1}\right)^{2}-\left(\chi^{2}\right)^{2}-\left(\chi^{3}\right)^{2}+\left(\chi^{4}\right)^{2}=l^{2}
$$

where $l$ is the so-called de Sitter length-parameter. We use the Latin alphabet $(a, b, c \ldots=0,1,2,3)$ to denote the four-dimensional algebra and tangent space indices, whose metric tensor is $\eta_{a b}=\operatorname{diag}(1,-1,-1,-1)$. Defining the dimensionless coordinate $\chi^{\prime 4}=\chi^{4} / l$, and using the notation $s=\eta_{44}$, the above conditions can be put together as

$$
\frac{1}{l^{2}} \eta_{a b} \chi^{a} \chi^{b}+\mathrm{s}\left(\chi^{4}\right)^{2}=\mathrm{s}
$$

For $s=-1$, we have the de Sitter space $d S=d S(4,1)$, whose metric is induced from the pseudo-Euclidean metric $\eta_{A B}$ $=(+1,-1,-1,-1,-1)$. It has the pseudo-orthogonal group $S O(4,1)$ as group of motions. Sign $\mathrm{s}=+1 \mathrm{corresponds}$ 
to anti-de Sitter space, denoted by $A d S=d S(3,2)$. It comes from $\eta_{A B}=(+1,-1,-1,-1,+1)$, and has $S O(3,2)$ as its group of motions. Both spaces are homogeneous [11]:

$$
d S=d S(4,1)=S O(4,1) / \mathscr{L} \quad \text { and } \quad A d S=d S(3,2)=S O(3,2) / \mathscr{L} .
$$

Furthermore, they are solutions of the sourceless Einstein's equation, provided the cosmological constant $\Lambda$ and the de Sitter length-parameter $l$ are related by

$$
\Lambda=-\frac{3 s}{l^{2}} .
$$

\subsection{Stereographic coordinates}

To comply with the observational data [12], we consider from now on the $d S$ case, for which $\Lambda \geq 0$ and

$$
-\frac{1}{l^{2}} \eta_{a b} \chi^{a} \chi^{b}+\left(\chi^{\prime 4}\right)^{2}=1
$$

The four-dimensional stereographic coordinates $\left\{x^{a}\right\}$ are obtained through a projection from the de Sitter hyper-surface into a target Minkowski spacetime. They are given by [13]

$$
\chi^{a}=\Omega(x) x^{a} \quad \text { and } \quad \chi^{4}=-\Omega(x)\left(1+\frac{\sigma^{2}}{4 l^{2}}\right)
$$

where

$$
\Omega(x)=\left(1-\frac{\sigma^{2}}{4 l^{2}}\right)^{-1}
$$

with $\sigma^{2}=\eta_{a b} x^{a} x^{b}$. The $\left\{x^{a}\right\}$ take values on the Minkowski spacetime on which the stereographic projection is made.

\subsection{Kinematic groups: transitivity}

In terms of the host-space Cartesian coordinates $\chi^{A}$, the generators of the infinitesimal de Sitter transformations are

$$
L_{A B}=\eta_{A C} \chi^{C} \frac{\partial}{\partial \chi^{B}}-\eta_{B C} \chi^{C} \frac{\partial}{\partial \chi^{A}},
$$

which satisfy the commutation relations

$$
\left[L_{A B}, L_{C D}\right]=\eta_{B C} L_{A D}+\eta_{A D} L_{B C}-\eta_{B D} L_{A C}-\eta_{A C} L_{B D} .
$$

In terms of the stereographic coordinates $\left\{x^{a}\right\}$, these generators are written as

$$
L_{a b}=\eta_{a c} x^{c} P_{b}-\eta_{b c} x^{c} P_{a}
$$

and

$$
L_{a 4}=l P_{a}-(4 l)^{-1} K_{a},
$$

where

$$
P_{a}=\partial / \partial x^{a}
$$

are the translation generators (with dimension of length ${ }^{-1}$ ), and

$$
K_{a}=\left(2 \eta_{a b} x^{b} x^{c}-\sigma^{2} \delta_{a}^{c}\right) P_{c}
$$

are the generators of proper conformal transformations (with dimension of length). The generators $L_{a b}$ refer to the Lorentz subgroup of de Sitter, whereas $L_{a 4}$ define transitivity on the corresponding de Sitter space. For this reason, they are usually called the de Sitter "translation" generators. The crucial point is to observe, as implied by Eq. (9), the de Sitter spacetime is transitive under a combination of translations and proper conformal transformations. The relative importance of each one of these transformations is determined by the value of the length parameter $l$, that is, by the value of the cosmological constant. 


\subsection{Contraction limits}

The group contraction procedure requires that, before each limit is taken, the generators be modified through an appropriate insertion of parameters. These alterations are frequently guided by dimensional considerations and are different for different limits [14]. For this reason, the $\Lambda \rightarrow 0$ and the $\Lambda \rightarrow \infty$ limits must be considered separately.

\subsubsection{Vanishing cosmological constant limit}

To study the limit of a vanishing cosmological constant $(l \rightarrow \infty)$, we rewrite the de Sitter generators in the forms

$$
L_{a b}=\eta_{a c} x^{c} P_{b}-\eta_{b c} x^{c} P_{a}
$$

and

$$
\Pi_{a} \equiv \frac{L_{a 4}}{l}=P_{a}-\frac{1}{4 l^{2}} K_{a} .
$$

The $L_{a b}$ 's generate the Lorentz transformations on Minkowski spacetime, and satisfy the commutation relation

$$
\left[L_{a b}, L_{c d}\right]=\eta_{b c} L_{a d}+\eta_{a d} L_{b c}-\eta_{b d} L_{a c}-\eta_{a c} L_{b d} .
$$

For $l \rightarrow \infty$, the generators $\Pi_{a}$ reduce to ordinary translations, and the de Sitter group contracts to the Poincare group $\mathscr{P}=\mathscr{L} \oslash \mathscr{T}$. Concomitant with the algebra and group deformations, the de Sitter space $d S(4,1)$ reduces to the Minkowski spacetime $M=\mathscr{P} / \mathscr{L}$, which is transitive under ordinary translations only.

\subsubsection{Infinite cosmological constant limit}

We remark, to begin with, that the limit $\Lambda \rightarrow \infty$ has to be understood as purely formal. In fact, considering that it corresponds to the small distance limit $l \rightarrow 0$, quantum effects should necessarily be taken into account. Such effects, as is well known, provide for $\Lambda$ a cutoff value which prevents the limit to be physically achieved. Actually, the reference value for defining small and large $\Lambda$ is the Planck cosmological constant $\Lambda_{P}=3 / l_{p}^{2}$, with $l_{P}$ the Planck length. A small $\Lambda$ will then be characterized by $\Lambda l_{P}^{2} \rightarrow 0$, and a large $\Lambda$ by $\Lambda l_{P}^{2} \rightarrow 1$.

The interest of the $\Lambda \rightarrow \infty$ limit lies in the fact that it yields the algebraic and geometric structures behind the "classical" physics of extremely high $\Lambda$. Let us then recall that the de Sitter space tends, in this case, to a cone spacetime, denoted $N$, which is related to Minkowski $M$ through the spacetime inversion [3]

$$
x^{a} \rightarrow-\frac{x^{a}}{\sigma^{2}} .
$$

By this inversion, the points at infinity on $M$ are led into the vertex of the cone-space $N$, and those on the light-cone of $M$ become the infinity of $N$. Applying it to the Minkowski interval

$$
d s^{2}=\eta_{a b} d x^{a} d x^{b}
$$

we see that ${ }^{1}$

$$
d s^{2} \rightarrow d \bar{s}^{2}=\bar{\eta}_{a b} d x^{a} d x^{b},
$$

where

$$
\bar{\eta}_{a b}=\sigma^{-4} \eta_{a b}, \quad \bar{\eta}^{a b}=\sigma^{4} \eta^{a b}
$$

is the metric on the cone-space $N$. It is important to recall also that the spacetime inversion (15) is well known to relate translations with proper conformal transformations [15]:

$$
P_{a} \rightarrow K_{a} .
$$

\footnotetext{
${ }^{1}$ In addition to denoting the indices of the Minkowski spacetime $M$, the Latin alphabet $(a, b, c \ldots=0,1,2,3)$ will also be used to denote the algebra and space indices of the cone spacetime $N$.
} 
On the other hand, it is found that the Lorentz generators do not change:

$$
L_{a b} \rightarrow L_{a b} .
$$

The above results imply that, to study the limit of an infinite cosmological constant $(l \rightarrow 0)$, it is necessary to write the de Sitter generators in the form

$$
\bar{L}_{a b} \equiv \sigma^{-4} L_{a b}=\bar{\eta}_{a c} x^{c} P_{b}-\bar{\eta}_{b c} x^{c} P_{a}
$$

and

$$
\bar{\Pi}_{a} \equiv 4 l L_{a 4}=4 l^{2} P_{a}-K_{a} .
$$

The generators $\bar{L}_{a b}$ satisfy the commutation relation

$$
\left[\bar{L}_{a b}, \bar{L}_{c d}\right]=\bar{\eta}_{b c} \bar{L}_{a d}+\bar{\eta}_{a d} \bar{L}_{b c}-\bar{\eta}_{b d} \bar{L}_{a c}-\bar{\eta}_{a c} \bar{L}_{b d} .
$$

Since $\bar{L}_{a b}$ satisfy Lorentz-like commutations relations, they can be interpreted as the generators of conformal Lorentz transformations, whose group we denote by $\overline{\mathscr{L}}$. For $l \rightarrow 0$, the generators $\bar{\Pi}_{a}$ reduce to (minus) the proper conformal generators, whose group we denote by $\overline{\mathscr{T}}$. In this limit, therefore, the de Sitter group contracts to the conformal Poincare group $\overline{\mathscr{P}}=\overline{\mathscr{L}} \oslash \overline{\mathscr{T}}$, the semi-direct product of the conformal Lorentz and the proper conformal groups [16]. As can easily be verified, the interval $d \bar{s}^{2}$ is invariant under $\overline{\mathscr{P}}$. Concomitant with the group contraction $l \rightarrow 0$, the de Sitter space reduces to the conic spacetime

$$
N=\overline{\mathscr{P}} / \overline{\mathscr{L}},
$$

which is transitive under proper conformal transformations.

\section{TRANSITIVITY AND THE NOTION OF DISTANCE}

The two concurrent, but different types of transformations present in the generators defining transitivity on the de Sitter spacetime $d S$ give rise to two different notions of distance: one which is related to translations, and another which is related to proper conformal transformations. This means that it is possible to define two different metrics on $d S$, one invariant under translations, and another invariant under proper conformal transformations. As a consequence, there will be two different family of geodesics, one joining all points equivalent under translations, and another joining all points equivalent under proper conformal transformations. If one considers only one of these families, therefore, there will be points on $d S$ which cannot be joined to each other by any geodesic. This is a well known property of the de Sitter spacetime [10]. In what follows we explore these concepts in more detail.

The Greek alphabet $(\mu, v, \rho, \ldots=0,1,2,3)$ will be used to denote indices related to the de Sitter spacetime. For example, its coordinates will be denoted by $\left\{x^{\mu}\right\}$. We recall that the Latin alphabet $(a, b, c \ldots=0,1,2,3)$ relate to the de Sitter algebra, as well as to the spacetime indices of both limits of the $d S$ spacetime: Minkowski space $(\Lambda \rightarrow 0$ limit) and the cone spacetime ( $\Lambda \rightarrow \infty$ limit). This allows the introduction of the holonomic tetrad $\delta^{a}{ }_{\mu}$, which satisfies

$$
\eta_{\mu v}=\delta^{a}{ }_{\mu} \delta^{b}{ }_{v} \eta_{a b}, \quad \bar{\eta}_{\mu v}=\delta^{a}{ }_{\mu} \delta^{b}{ }_{v} \bar{\eta}_{a b} .
$$

Consequently, we can also write

$$
\sigma^{2}=\eta_{a b} x^{a} x^{b}=\eta_{\mu \nu} x^{\mu} x^{\nu}, \quad \bar{\sigma}^{2}=\bar{\eta}_{a b} x^{a} x^{b}=\bar{\eta}_{\mu \nu} x^{\mu} x^{\nu},
$$

where we have identified $x^{a}=\delta^{a}{ }_{\mu} x^{\mu}$.

\subsection{Translational distance}

The first notion of distance is that related to translations, which become the dominant part of the $d S$ transitivity generators for small values of $\Lambda$. To study its properties it is, therefore, necessary to use a parameterization appropriate for the limit $\Lambda \rightarrow 0$. This parameterization is naturally provided by Eq. (1),

$$
K_{G} \Omega^{2}(x) \sigma^{2}+\left(\chi^{\prime 4}\right)^{2}=1,
$$


where the Gaussian curvature

$$
K_{G}=-1 / l^{2}
$$

of the $d S$ spacetime turns up. We introduce now the anholonomic tetrad field

$$
h^{a}{ }_{\mu}=\Omega \delta^{a}{ }_{\mu}
$$

If $\eta_{a b}$ denotes the Minkowski metric, the de Sitter metric can, in this case, be written as

$$
g_{\mu \nu} \equiv h^{a}{ }_{\mu} h^{b}{ }_{v} \eta_{a b}=\Omega^{2}(x) \eta_{\mu \nu}
$$

It defines the "translational distance", with squared interval

$$
d \tau^{2}=g_{\mu \nu} d x^{\mu} d x^{v} \equiv \Omega^{2}(x) \eta_{\mu \nu} d x^{\mu} d x^{v}
$$

For $l \rightarrow \infty(\Lambda \rightarrow 0), d S$ contracts to the Minkowski spacetime $M$, and $d \tau^{2}$ reduces to the Lorentz-invariant interval

$$
d \tau^{2} \rightarrow d s^{2}=\eta_{\mu v} d x^{\mu} d x^{v}
$$

Due to its translational transitivity, this is the only notion of distance that can be defined on $M$.

\subsection{Conformal distance}

The second notion of distance is that related to the proper conformal transformation. Since this transformation is the most important part of the transitivity generators for large values of $\Lambda$, its study requires a parameterization appropriate for the limit $\Lambda \rightarrow \infty$. This can be achieved by rewriting Eq. (1) in the form

$$
\bar{K}_{G} \bar{\Omega}^{2}(x) \bar{\sigma}^{2}+\left(\chi^{\prime 4}\right)^{2}=1
$$

where

$$
\bar{\Omega}(x) \equiv \frac{\sigma^{2}}{4 l^{2}} \Omega(x)=-\frac{1}{\left(1-4 l^{2} / \sigma^{2}\right)}
$$

is the new conformal factor, and

$$
\bar{K}_{G}=-16 l^{2}
$$

is the conformal Gaussian curvature. We introduce now the anholonomic tetrad field

$$
\bar{h}_{\mu}^{a}=\bar{\Omega}(x) \delta_{\mu}^{a} .
$$

If $\bar{\eta}_{a b}$ denotes the cone spacetime metric, the corresponding de Sitter metric can, in this case, be written as

$$
\bar{g}_{\mu \nu} \equiv \bar{h}_{\mu}^{a} \bar{h}^{b}{ }_{\nu} \bar{\eta}_{a b}=\bar{\Omega}^{2}(x) \bar{\eta}_{\mu \nu}
$$

It defines the "conformal distance" on de Sitter spacetime, whose quadratic interval has the form

$$
d \bar{\tau}^{2} \equiv \bar{g}_{\mu \nu} d x^{\mu} d x^{\nu}=\bar{\Omega}^{2}(x) \bar{\eta}_{\mu \nu} d x^{\mu} d x^{\nu}
$$

For $l \rightarrow 0(\Lambda \rightarrow \infty), d S$ contracts to the cone spacetime $N$, and $d \bar{\tau}^{2}$ reduces to the conformal invariant interval on $N$ :

$$
d \bar{\tau}^{2} \rightarrow d \bar{s}^{2}=\bar{\eta}_{\mu v} d x^{\mu} d x^{v}
$$

On account of the conformal transitivity of the cone spacetime, this is the only notion of distance that can be defined on $N[17]$. 


\subsection{Two family of geodesics}

The Christoffel connection of the de Sitter spacetime metric $g_{\mu \nu}$, given by Eq. (29), is

$$
\Gamma_{\mu \nu}^{\lambda}=\left[\delta^{\lambda}{ }_{\mu} \delta^{\sigma}{ }_{\nu}+\delta^{\lambda}{ }_{\nu} \delta^{\sigma}{ }_{\mu}-\eta_{\mu \nu} \eta^{\lambda \sigma}\right] \partial_{\sigma}[\ln \Omega(x)]
$$

The corresponding Riemann tensor components are

$$
R^{\mu}{ }_{v \rho \sigma}=-\frac{1}{l^{2}}\left[\delta_{\rho}^{\mu} g_{v \sigma}-\delta_{\sigma}^{\mu} g_{v \rho}\right] .
$$

As already remarked, there are points in the de Sitter spacetime which cannot be connected by any geodesic defined by the metric connection (39). The reason for this fact is that the metric $g_{\mu \nu}$ defines a "translational distance" only, whereas the de Sitter spacetime is homogeneous under a combination of translation and proper conformal transformations.

On the other hand, the Christoffel connection of the de Sitter spacetime $\bar{g}_{\mu v}$, given by Eq. (36), is

$$
\bar{\Gamma}_{\mu v}^{\lambda}=\left[\delta_{\mu}^{\lambda} \delta^{\sigma}{ }_{\nu}+\delta^{\lambda}{ }_{\nu} \delta^{\sigma}{ }_{\mu}-\bar{\eta}_{\mu \nu} \bar{\eta}^{\lambda \sigma}\right] \partial_{\sigma}[\ln \bar{\Omega}(x)]
$$

The corresponding Riemann tensor components are

$$
\bar{R}_{v \rho \sigma}^{\mu}=-16 l^{2}\left[\delta_{\rho}^{\mu} \bar{g}_{v \sigma}-\delta^{\mu} \bar{g}_{v \rho}\right] .
$$

Since the metric $\bar{g}_{\mu \nu}$ defines only a "conformal distance", and since the de Sitter spacetime is homogeneous under a combination of translation and proper conformal transformations, there will again be points on $d S$ which cannot be connected by any of the geodesics defined by the metric connection (41). However, the two families of geodesics are complementary in the sense that the points that cannot be connected by one family of geodesics can be connected by the other family. In other words, any two points of the de Sitter spacetime can be connected by a geodesics belonging to one or another of the two families of geodesics.

It is important to remark that both Riemann tensors $R^{\mu}{ }_{v \rho \sigma}$ and $\bar{R}^{\mu}{ }_{v \rho \sigma}$ represent the curvature of the de Sitter spacetime. The difference is that, whereas $R^{\mu}{ }_{v \rho \sigma}$ represents the curvature tensor in a parameterization appropriate for the limit of a vanishing cosmological constant, $\bar{R}^{\mu}{ }_{v \rho \sigma}$ represents the curvature tensor in a parameterization suitable for the limit of an infinite cosmological constant. As a straightforward calculation shows, both limits yield a spacetime with vanishing curvature: both Minkowski and the cone spacetimes are flat.

\section{DE SITTER SPECIAL RELATIVITY}

\subsection{The de Sitter transformations}

The de Sitter transformations can be thought of as rotations in a five-dimensional pseudo-Euclidian spacetime,

$$
\chi^{\prime C}=\Lambda_{D}^{C} \chi^{D}
$$

with $\Lambda^{C}{ }_{D}$ the group element in the vector representation. Since these transformations leave invariant the quadratic form

$$
-\eta_{A B} \chi^{A} \chi^{B}=l^{2}
$$

they also leave invariant the length parameter $l$. Their infinitesimal form is

$$
\delta \chi^{C}=\frac{1}{2} \mathscr{E}^{\mathscr{A} B} L_{A B} \chi^{C}
$$

where $\mathscr{E}^{A B}$ are the parameters and $L_{A B}$ the generators. 


\subsubsection{Small cosmological constant}

For $\Lambda$ small, analogously to the identifications (12) and (13), we define the parameters

$$
\varepsilon^{a b}=\mathscr{E}^{a b} \quad \text { and } \quad \varepsilon^{a}=l \mathscr{E}^{a 4}
$$

In this case, in terms of the stereographic coordinates, the infinitesimal de Sitter transformation assumes the form

$$
\delta x^{c}=\frac{1}{2} \varepsilon^{a b} L_{a b} x^{c}+\varepsilon^{a} \Pi_{a} x^{c},
$$

or equivalently

$$
\delta x^{c}=\varepsilon_{a}^{c} x^{a}+\varepsilon^{a}-\frac{\varepsilon^{b}}{4 l^{2}}\left(2 x_{b} x^{c}-\sigma^{2} \delta_{b}{ }^{c}\right) .
$$

In the limit of a vanishing $\Lambda$, it reduces to the ordinary Poincaré transformation.

\subsubsection{Large cosmological constant}

For $\Lambda$ large, analogously to the identifications (21) and (22), we define the parameters

$$
\bar{\varepsilon}^{a b}=\sigma^{4} \mathscr{E} a b \quad \text { and } \quad \bar{\varepsilon}^{a}=\mathscr{E}^{a 4} / 4 l .
$$

In this case, in terms of the stereographic coordinates, the de Sitter transformation assumes the form

$$
\delta x^{c}=\frac{1}{2} \bar{\varepsilon}^{a b} \bar{L}_{a b} x^{c}+\bar{\varepsilon}^{a} \bar{\Pi}_{a} x^{c},
$$

or equivalently

$$
\delta x^{c}=\bar{\varepsilon}_{a}^{c} x^{a}-\bar{\varepsilon}^{a}\left(2 x_{b} x^{c}-\sigma^{2} \delta_{b}^{c}\right)+4 l^{2} \bar{\varepsilon}^{a}
$$

where $\bar{\varepsilon}_{a}^{c}=\bar{\varepsilon}^{c b} \bar{\eta}_{b a} \equiv \varepsilon^{c}{ }_{a}$. In the limit of an infinite $\Lambda$, it reduces to the a conformal Poincare transformation.

\subsection{The Lorentz generators}

Up to now, we have studied the de Sitter transformations on a Minkowski space. In what follows we are going to study the form of the corresponding generators on a de Sitter spacetime, which is the underlying spacetime of a de Sitter special relativity. This will be done by contracting the generators acting in Minkowski spacetime with the appropriate tetrads. We begin by considering the Lorentz generators.

\subsubsection{Small cosmological constant}

For small $\Lambda$, the generators of an infinitesimal Lorentz transformation are (see section 4.1.1)

$$
L_{a b}=\eta_{a c} x^{c} P_{b}-\eta_{b c} x^{c} P_{a}
$$

The corresponding generators acting on a de Sitter spacetime can be obtained by contracting $L_{a b}$ with the tetrad $h^{a} \mu$ given by Eq. (28):

$$
\mathscr{L}_{\mu v} \equiv h^{a}{ }_{\mu} h^{b}{ }_{v} L_{a b}=g_{\mu \rho} x^{\rho} P_{v}-g_{\nu \rho} x^{\rho} P_{\mu} .
$$

These generators are easily found to satisfy the commutation relations

$$
\left[\mathscr{L}_{\mu \nu}, \mathscr{L}_{\rho \lambda}\right]=g_{v \rho} \mathscr{L}_{\mu \lambda}+g_{\mu \lambda} \mathscr{L}_{v \rho}-g_{v \lambda} \mathscr{L}_{\mu \rho}-g_{\mu \rho} \mathscr{L}_{v \lambda}
$$

Even when acting on de Sitter spacetime, therefore, these generators still present a well-defined algebraic structure, isomorphic to the usual Lie algebra of the Lorentz group. This is a fundamental property in the sense that it allows the construction, on the de Sitter spacetime, of an algebraically well defined special relativity. This possibility is related to the mentioned fact that, like the Minkowski spacetime, the de Sitter spacetime is homogeneous and isotropic [18]. 


\subsubsection{Large cosmological constant}

For $\Lambda$ large, the generators of infinitesimal Lorentz transformations are (see section 4.1.2)

$$
\bar{L}_{a b}=\bar{\eta}_{a c} x^{c} P_{b}-\bar{\eta}_{b c} x^{c} P_{a} .
$$

On a de Sitter spacetime, their explicit form can be obtained by contracting (55) with the tetrad $\bar{h}^{a}{ }_{\mu}$ of Eq. (35):

$$
\overline{\mathscr{L}}_{\mu v} \equiv \bar{h}^{a}{ }_{\mu} \bar{h}^{b}{ }_{v} \bar{L}_{a b}=\bar{g}_{\mu \rho} x^{\rho} P_{v}-\bar{g}_{v \rho} x^{\rho} P_{\mu} .
$$

These generators are easily found to satisfy the commutation relations

$$
\left[\overline{\mathscr{L}}_{\mu v}, \overline{\mathscr{L}}_{\rho \lambda}\right]=\bar{g}_{v \rho} \overline{\mathscr{L}}_{\mu \lambda}+\bar{g}_{\mu \lambda} \overline{\mathscr{L}}_{\nu \rho}-\bar{g}_{v \lambda} \overline{\mathscr{L}}_{\mu \rho}-\bar{g}_{\mu \rho} \overline{\mathscr{L}}_{v \lambda}
$$

Like $\mathscr{L}_{\mu v}$, therefore, they present a Lorentz-like algebraic structure.

\subsection{The de Sitter "translation" generators}

Like in the case of the Lorentz generators, the form of the de Sitter "translation" generators acting in the de Sitter spacetime can be obtained by contracting $\Pi^{a}$ and $\bar{\Pi}^{a}$ with the appropriate tetrad.

\subsubsection{Small cosmological constant}

For $\Lambda$ small, the de Sitter translation generators are given by

$$
\Pi_{\mu} \equiv h^{a}{ }_{\mu} \Pi^{a}=\Omega\left[P_{\mu}-\left(1 / 4 l^{2}\right) K_{\mu}\right],
$$

where

$$
P_{\mu}=\partial / \partial x^{\mu} \quad \text { and } \quad K_{\mu}=\left(2 \eta_{\mu \rho} x^{\rho} x^{v}-\sigma^{2} \delta_{\mu}^{v}\right) P_{v}
$$

\subsubsection{Large cosmological constant}

For $\Lambda$ large, on the other hand, they are

$$
\bar{\Pi}_{\mu} \equiv \bar{h}^{a}{ }_{\mu} \bar{\Pi}^{a}=\bar{\Omega}\left[\left(4 l^{2}\right) P_{\mu}-K_{\mu}\right] .
$$

We see from these expressions that the de Sitter spacetime is transitive under a combination of the translation and proper conformal generators. For $\Lambda \rightarrow 0, \Pi_{\mu}$ reduce to the usual translation generators of Minkowski spacetime. For $\Lambda \rightarrow \infty, \bar{\Pi}_{\mu}$ reduce to (minus) the proper conformal generators, which define the transitivity on the cone spacetime.

\section{ENERGY-MOMENTUM RELATIONS}

Let us consider now the mechanics of structureless particles on de Sitter spacetime. The conserved Noether current associated to a particle of mass $m$ is, in this case, the five-dimensional angular momentum [13]

$$
\lambda^{A B}=m c\left(\chi^{A} \frac{d \chi^{B}}{d \tau}-\chi^{B} \frac{d \chi^{A}}{d \tau}\right),
$$

with $d \tau$ the de Sitter line element (30). In order to make contact with the usual definitions of energy and momentum, we rewrite it in terms of the stereographic coordinates $\left\{x^{a}\right\}$ and the Minkowski interval $d s$. The result is

$$
\lambda^{a b}=x^{a} p^{b}-x^{b} p^{a}
$$


and

$$
\lambda^{a 4}=l p^{a}-(4 l)^{-1} k^{a}
$$

where

is the momentum, and

$$
p^{a}=m c \Omega \frac{d x^{a}}{d s}
$$

$$
k^{a}=\left(2 \eta_{c b} x^{c} x^{a}-\sigma^{2} \delta_{b}{ }^{a}\right) p^{b}
$$

is the so-called conformal momentum [15]. Their form on the de Sitter spacetime can be obtained through a contraction with appropriate tetrads.

\subsection{Small cosmological constant}

For $\Lambda l_{P}^{2} \rightarrow 0$, analogously to the generators, we define the de Sitter momentum

$$
\pi^{a} \equiv \frac{\lambda^{a 4}}{l}=p^{a}-\frac{k^{a}}{4 l^{2}} .
$$

The corresponding spacetime version is

$$
\pi^{\mu} \equiv h_{a}{ }^{\mu} \pi^{a}=p^{\mu}-\frac{k^{\mu}}{4 l^{2}}
$$

where

is the Poincaré momentum, and

$$
p^{\mu}=m c \frac{d x^{\mu}}{d s}
$$

$$
k^{\mu}=\left(2 \eta_{\lambda \rho} x^{\rho} x^{\mu}-\sigma^{2} \delta_{\lambda}{ }^{\mu}\right) p^{\lambda}
$$

is the corresponding conformal Poincaré momentum. We remark that $\pi^{\mu}$ is the conserved Noether momentum related to the transformations generated by $\Pi_{a}$. Similarly to the identification $p^{\mu}=T^{\mu 0}$, with $T^{\mu v}$ the energy-momentum current, the conformal momentum $k^{\mu}$ is defined by $k^{\mu}=K^{\mu 0}$, with $K^{\mu v}$ the conformal current [15].

If we define the de Sitter mass $M$ as the first Casimir invariant of the de Sitter group [13]

$$
g_{\mu \nu} \pi^{\mu} \pi^{v} \equiv M^{2} c^{2}
$$

and write the components of $\pi^{\mu}$ as $(i, j, \ldots=1,2,3)$

$$
\pi^{\mu} \equiv\left(\frac{E}{c}, P^{i}\right)
$$

with $E$ and $P^{i}$ the de Sitter notions of energy and momentum, the kinematic relation in the de Sitter relativity can be written in the form

$$
\frac{E^{2}}{c^{2}}=P^{2}=M^{2} c^{2} .
$$

However, in order to make contact with ordinary special relativity, it is convenient to rewrite the dispersion relation (72) in terms of the usual notions of mass, energy and momentum. To do it, we observe first that, whereas

$$
\pi^{0} \equiv p^{0}-\frac{k^{0}}{4 l^{2}}
$$

represents the energy, the space components

$$
\pi^{i} \equiv p^{i}-\frac{k^{i}}{4 l^{2}}
$$

represent the momentum. The presence of a cosmological constant, therefore, changes the usual definitions of energy and momentum $[4,19]$. When written in terms of these usual notions, the energy-momentum relation (70) becomes

$$
g_{\mu \nu} \pi^{\mu} \pi^{v}=\Omega^{2} \eta_{\mu v}\left(p^{\mu} p^{\nu}-\frac{1}{2 l^{2}} p^{\mu} k^{\nu}+\frac{1}{16 l^{4}} k^{\mu} k^{v}\right) .
$$


The components of the Poincare momentum $p^{\mu}$ are

$$
p^{\mu}=\left(\frac{\varepsilon_{p}}{c}, p^{i}\right)
$$

where $\varepsilon_{p}$ and $p^{i}$ are the usual Poincaré energy and momentum, respectively, related by $\eta_{\mu v} p^{\mu} p^{\nu}=m^{2} c^{2}$, where $m^{2} c^{2}$ is the first Casimir invariant of the Poincare group. Analogously, the components of the conformal momentum $k^{\mu}$ can be written in the form

$$
k^{\mu}=\left(\frac{\varepsilon_{k}}{c}, k^{i}\right)
$$

with $\varepsilon_{k}$ the conformal notion of energy, and $k^{i}$ the space components of the conformal momentum. The conformal momentum satisfies $\eta_{\mu v} k^{\mu} k^{v}=\bar{m}^{2} c^{2}$, where $\bar{m}^{2} c^{2}$ is the first Casimir invariant of the conformal Poincare group, with $\bar{m}^{2}=\sigma^{4} m^{2}$ the conformal equivalent of the mass [1]. Using the expressions above, the relation (75) becomes

$$
\frac{\varepsilon_{p}^{2}}{c^{2}}-p^{2}=m^{2} c^{2}+\frac{1}{2 l^{2}}\left[\frac{\varepsilon_{p} \varepsilon_{k}}{c^{2}}-\vec{p} \cdot \vec{k}-m \bar{m} c^{2}-\frac{1}{8 l^{2}}\left(\frac{\varepsilon_{k}^{2}}{c^{2}}-k^{2}-\bar{m}^{2} c^{2}\right)\right] .
$$

For small values of $\Lambda$, the de Sitter length parameter $l$ is large, and the modifications in the energy-momentum relation will be small. Up to first order in $\Lambda$, we get

$$
\frac{\varepsilon_{p}^{2}}{c^{2}}-p^{2} \simeq m^{2} c^{2}+\frac{1}{2 l^{2}}\left[\frac{\varepsilon_{p} \varepsilon_{k}}{c^{2}}-\vec{p} \cdot \vec{k}-m \bar{m} c^{2}\right] .
$$

In the limit of a vanishing cosmological constant, the ordinary notions of energy and momentum are recovered, and the de Sitter relativity reduces to the ordinary special relativity, in which the Poincare symmetry is exact. The energymomentum relation, in this case, reduces to the usual expression

$$
\frac{\varepsilon_{p}^{2}}{c^{2}}-p^{2}=m^{2} c^{2}
$$

\subsection{Large cosmological constant}

For $\Lambda l_{P}^{2} \rightarrow 1$, analogously to the generators, we define the de Sitter momentum

$$
\pi^{a} \equiv 4 l \lambda^{a 4}=4 l^{2} p^{a}-k^{a} .
$$

The corresponding spacetime version is

$$
\bar{\pi}^{\mu} \equiv \bar{h}_{a}^{\mu} \bar{\pi}^{a}=\frac{4 l^{2}}{\sigma^{2}}\left(4 l^{2} p^{\mu}-k^{\mu}\right) .
$$

We remark that $\bar{\pi}^{\mu}$ is the conserved Noether momentum related to the transformations generated by $\bar{\Pi}_{a}$. Defining the de Sitter conformal mass $\bar{M}$ by

$$
\bar{g}_{\mu v} \bar{\pi}^{\mu} \bar{\pi}^{v} \equiv \bar{M} c^{2}
$$

and writing the components of $\bar{\pi}^{\mu}$ as

$$
\bar{\pi}^{\mu} \equiv\left(\frac{\bar{E}}{c}, \bar{K}^{i}\right)
$$

with $\bar{E}$ and $\bar{K}^{i}$ the de Sitter notions of conformal energy and conformal momentum, the kinematic relation in this case assumes the form

$$
\frac{\bar{E}^{2}}{c^{2}}=\bar{K}^{2}+\bar{M}^{2} c^{2}
$$

Like in the previous case, it is convenient to rewrite the dispersion relation (85) ini terms of the usual notions of conformal mass, energy and momentum. To do it, we observe that, whereas

$$
\bar{\pi}^{0}=\frac{4 l^{2}}{\sigma^{2}}\left(4 l^{2} p^{0}-k^{0}\right)
$$


represents the conformal energy, the space components

$$
\bar{\pi}^{i}=\frac{4 l^{2}}{\sigma^{2}}\left(4 l^{2} p^{i}-k^{i}\right)
$$

represent the conformal momentum. In this case, the energy-momentum relation (83) becomes

$$
\bar{g}_{\mu \nu} \bar{\pi}^{\mu} \bar{\pi}^{v}=16 l^{4} \bar{\Omega}^{2} \sigma^{-8} \eta_{\mu \nu}\left[16 l^{4} p^{\mu} p^{\nu}-8 l^{2} p^{\mu} k^{v}+k^{\mu} k^{v}\right]
$$

or equivalently,

$$
\frac{\varepsilon_{k}^{2}}{c^{2}}-k^{2}=\bar{m}^{2} c^{2}+8 l^{2}\left[\frac{\varepsilon_{p} \varepsilon_{k}}{c^{2}}-\vec{p} \cdot \vec{k}-m \bar{m} c^{2}-2 l^{2}\left(\frac{\varepsilon_{p}^{2}}{c^{2}}-p^{2}-m^{2} c^{2}\right)\right] .
$$

For large values of the cosmological constant, the de Sitter length parameter $l$ is small. Up to first order in $l^{2}$, we get

$$
\frac{\varepsilon_{k}^{2}}{c^{2}}-k^{2} \simeq \bar{m}^{2} c^{2}+8 l^{2}\left[\frac{\varepsilon_{p} \varepsilon_{k}}{c^{2}}-\vec{p} \cdot \vec{k}-m \bar{m} c^{2}\right] .
$$

In the formal limit $\Lambda l_{P}^{2} \rightarrow \infty$, only the conformal notions of energy and momentum will remain, and de Sitter relativity will reduce to the pure conformal special relativity. In this case, the energy-momentum relation acquires the conformal special relativistic form

$$
\frac{\varepsilon_{k}^{2}}{c^{2}}-k^{2}=\bar{m}^{2} c^{2}
$$

\section{FINAL REMARKS}

A non-vanishing cosmological term introduces the conformal generators in the definition of spacetime transitivity. As a consequence, the conformal transformations will naturally be incorporated in the kinematics of spacetime, and the corresponding conformal current will appear as part of the Noether conserved current. Of course, for a small enough cosmological term, the conformal modifications become negligible and ordinary physics remains valid. For large values of $\Lambda$, however, the conformal contributions to the physical magnitudes cannot be neglected, and these contributions will give rise to deep conceptual changes. For example, ordinary special relativity, which is based on the Poincaré group, will no longer be true, and must be replaced by a new special relativity based on the de Sitter group. The physical tangent space at each point of any spacetime will consequently be converted into an osculating de Sitter spacetime.

Due to the fact that the de Sitter spacetime is transitive under a combination of translation and proper conformal transformations, the de Sitter special relativity can be viewed as made up of two different relativities: the usual one, related to translations, and a conformal one, related to proper conformal transformations. It is a single relativity interpolating these two extreme limiting cases. For small values of $\Lambda$, ordinary special relativity will prevail over the conformal one, and the Poincaré symmetry will be weakly deformed. In the contraction limit of a vanishing $\Lambda$, de Sitter relativity reduces to the ordinary special relativity, and the underlying spacetime reduces to the Minkowski space $M$, which is transitive under translations. For large values of $\Lambda$, on the other hand, conformal relativity will prevail over the usual one, and the Poincare symmetry will be strongly deformed. In the contraction limit of an infinite $\Lambda$, de Sitter special relativity reduces to a conformal relativity, and the underlying spacetime reduces to a maximally symmetric cone spacetime, which is transitive under proper conformal transformations only. Although translations, and consequently the usual notion of distance, cannot be defined on such spacetime, it can be said to be conformally infinite. Physically, the cone spacetime represents a universe in which all energy is in the form of dark energy [3].

\subsection{Relation to doubly special relativity}

The de Sitter group can be interpreted as a particular deformation of the Poincare group. It is related to Poincaré through the contraction limit of a vanishing cosmological constant, in the very same way the Galilei group is related to the Poincare group through the contraction limit of an infinite velocity of light. A special relativity based on the de Sitter group, therefore, gives rise to a specific kind of deformed (or doubly, as it has been called) special relativity 
(DSR) [20]. In the usual approach to DSR, the Poincare symmetry is deformed through the agency of a dimensional parameter $\kappa$, proportional to the Planck length. Such a deformation implies that, in the high energy limit, the new ensuing relativity principle requires that physics become invariant, not under Poincaré, but under a " $\kappa$-deformed" Poincare group, which reduces to the standard Poincare group in the low energy limit.

A crucial difference between the standard formulations of DSR and a de Sitter special relativity concerns the structure of the symmetry groups. In a $\kappa$-deformed Poincare group, the Lorentz subgroup is deformed by the $\kappa$ parameter, and consequently the corresponding relativity will include a violation of the Lorentz symmetry at the scales where the theory is supposed to hold up. On the other hand, in a de Sitter special relativity, the Lorentz subgroup remains untouched, and consequently the Lorentz symmetry remains a true symmetry at all scales. Instead of Lorentz, the presence of a non-vanishing $\Lambda$ produces a violation of the translation symmetry. In fact, when Minkowski spacetime is replaced by de Sitter, the corresponding kinematic groups change from Poincaré to de Sitter. Since, algebraically speaking, the only difference between these two groups is the replacement of $P_{a}$ by a linear combination of $P_{a}$ and $K_{a}$, the net result of this change is ultimately the breakdown of translational invariance. From the experimental point of view, therefore, a de Sitter special relativity could be probed by looking for possible violations of translational invariance. This could be done, for example, by applying the same techniques used in the search for possible violations of Lorentz and CPT symmetries in high energy processes [21]. For small values of $\Lambda$, as we have seen, the homogeneity of spacetime is preponderantly defined by the translation generators, which means that the violation of the translation invariance will be very small. Only when $\Lambda$ is large this violation is expected to be relevant.

Another important difference is that, in the standard $\kappa$-deformed formulations of doubly special relativity, although energy and momentum keep their ordinary special relativistic notions, they are assumed to satisfy a deformed dispersion relation. As a consequence, a consistent notion of total energy and momentum, as well as a conservation law for them, is lacking in these theories [22]. In a de Sitter special relativity, on the other hand, a modified but precise notion of momentum and energy is provided: they are the Noether currents associated to the de Sitter symmetry. There is a clear relation between the symmetry generators (8-9) and the conserved currents (62-63). The resulting deformed dispersion relations for the particle's energy and momentum, given by Eqs. (78) and (89), are consequently relations between conserved quantities. Since the de Sitter current is a linear combination of the ordinary momentum $p^{\mu}$ and the conformal momentum $k^{\mu}$, the dispersion relations turn out to depend on these two four-vectors. Notice, however, that neither $p^{\mu}$ nor $k^{\mu}$ is conserved: only the de Sitter momentum is conserved.

It is also worth mentioning that, in the very same way as it happens with the ordinary special relativity dispersion relation (80), the de Sitter dispersion relations (78) and (89) are invariant under a simultaneous re-scaling of mass, energy and momentum. On the other hand, because it includes non-quadratic terms in the momentum, the dispersion relations of the usual formulations of DSR are not invariant under such a re-scaling. As a consequence, they present the so called "soccer-ball problem" [23]. This is not the case of the dispersion relations of the de Sitter special relativity, whose scale invariance make them true for elementary particles (as it is argued since they must be relevant for discussing elementary particle processes), as well as for macroscopic objects like, for example, a soccer-ball.

\subsection{Causal structure and quantum gravity}

If our current theories of particle physics based on spontaneously broken symmetry and phase transition are correct, there must have been some periods in the history of the Universe in which the value of $\Lambda$, and hence of the scale energy $E_{\Lambda}$, were large. For example, in the electroweak epoch characterized by $\Lambda_{E W}$, the kinematics of a typical electroweak process with energy $E_{\Lambda_{E W}}$, according to the de Sitter special relativity, must have been strongly influenced by $\Lambda_{E W}$. In fact, as we have seen, a large cosmological constant would produce significant changes in the definitions of energy and momentum, as well as in the kinematic relations satisfied by them. These changes could modify significantly the physics that should be applied in the study the early universe. To understand these changes, it is important to notice that the conformal symmetry of high- $\Lambda$ spacetimes is not just a symmetry presented by some physical systems, like for example the conformal symmetry of the electromagnetic field, but a kinematic symmetry, that is, a symmetry related to the relativity principle governing the physics at that scale.

On the other hand, if we take spontaneously broken symmetries as the primary source of a non-vanishing $\Lambda$, it is conceivable that a high energy experiment could modify the local structure of space-time for a short period of time, in such a way that the immediate neighborhood of a high energy collision would depart from the Minkowski space and become a de Sitter spacetime. According to this scheme, there would be a connection between the energy scale of the experiment and the local value of $\Lambda$ [24]. For energies of the order of $200 \mathrm{GeV}$, corresponding to the electroweak 
phase transition, the de Sitter parameter is $l_{\Lambda_{E W}} \sim 0.25 \mathrm{~cm}$, which is equivalent to $E_{\Lambda_{E W}} \sim 10^{-4} \mathrm{eV}$. For high energy experiments of order $20 \mathrm{TeV}$, one finds $E_{\Lambda_{T e V}} \sim 1 \mathrm{eV}$. And, for energies of order $1000 \mathrm{TeV}$, we have $E_{\Lambda} \sim 2500 \mathrm{eV}$. For particles of small mass, such as neutrinos, there would be significant changes in the kinematics at very high energies, which could eventually be tested in the existing or planned colliders [24].

Another important point refers to some possible implications to quantum gravity. Due to the homogeneous character of the de Sitter spacetime, the Lorentz generators in this spacetime still present a well defined algebraic structure, isomorphic to the usual Lie algebra of the Minkowski Lorentz group. This means that the Lorentz symmetry remains a sub-symmetry in a de Sitter relativity, and consequently the velocity of light $c$ is left unchanged by a de Sitter transformation. Since it also leaves unchanged the length parameter $l$, a de Sitter transformation leaves unchanged both $c$ and $l$. This property has important consequences for causality. As is well known, the constancy of $c$ introduces a causal structure in spacetime, defined by the light cones. The presence of the de Sitter length parameter $l$ adds to that structure some further restrictions on the causal structure of spacetime. To see that, let us remember that the de Sitter spacetime has a horizon, which restricts the causal region of each observer. In terms of the stereographic coordinates, this horizon is identified by

$$
x^{2}+y^{2}+z^{2}=l^{2} / \Omega^{2} \text { and }\left(x^{0}\right)^{2}=l^{2}(2-1 / \Omega)^{2} .
$$

For small $\Lambda$, the horizon tends to infinity, and there are no significant causal changes. For large values of $\Lambda$, however, the causal domain of each observer — restricted by the horizon - becomes small. Considering again a possible $\Lambda$ dependence of high energy processes, for experiments of the order of the Planck energy, this region would be of the order of the Planck length $l_{P}$. At this scale, therefore, the large value of $\Lambda$ would introduce deep changes in the causal structure of spacetime [25]. In particular, a de Sitter transformation, at this scale, would leave both $c$ and $l_{P}$ unchanged. Finally, it is interesting to observe that, since the area $A_{d S}$ of the de Sitter horizon is proportional to $l^{2}$, the entropy associated to this surface will be proportional to the logarithm of the number of states

$$
n=A_{d S} / l_{P}^{2} \sim l^{2} / l_{P}^{2}
$$

At the Planck scale, $n \sim 1$, which yields a vanishing entropy for the de Sitter horizon. This property provides a contact between de Sitter special relativity and quantum gravity [26], and could be important for the understanding of the spacetime structure at the Planck scale.

\section{ACKNOWLEDGMENTS}

The authors would like to thank M. Novello for the invitation to present this lecture at the School. They would also like to thank FAPESP, CNPq and CAPES for financial support.

\section{REFERENCES}

1. R. Aldrovandi, J. P. Beltrán Almeida and J. G. Pereira, Class. Quant. Grav. (2007), in press (Preprint gr-qc/0606122).

2. H. Y. Guo, C. G. Huang, Z. Xu and B. Zhuo, Phys. Lett. A 331, 1 (2204) (Preprint hep-th/0403171).

3. R. Aldrovandi, J. P. Beltrán Almeida and J. G. Pereira, J. Geom. Phys 56, 1042 (2006) (Preprint gr-qc/0403099).

4. R. Aldrovandi, J. P. Beltrán Almeida and J. G. Pereira, Int. J. Mod. Phys. D 13, 2241 (2004) (Preprint gr-qc/0405104).

5. E. Inönü and E. P. Wigner, Proc. Natl. Acad. Scien. 39, 510 (1953).

6. R. Gilmore, Lie Groups, Lie Algebras, and Some of Their Applications, Wiley, New York, 1974.

7. H. Bacry and J.-M Lévy-Leblond, J. Math. Phys. 9, 1605 (1968); C. Duval, G. Burdet, H. P. Künsle and M. Perrin, Phys. Rev. D 31, 1841 (1985); R. Aldrovandi, A. L. Barbosa, L. C. B. Crispino and J. G. Pereira, Class. Quant. Grav. 16, 495 (1999) (Preprint gr-qc/9801100).

8. G. W. Gibbons and C. E. Patricot, Class. Quant. Grav. 20, 5225 (2003) (Preprint hep-th/0308200).

9. S. Weinberg, Gravitation and Cosmology, Wiley, New York, 1972.

10. S. W. Hawking and G. F. R Ellis, The Large Scale Structure of Space-Time, Cambridge University Press, Cambridge, 1973.

11. R. Aldrovandi and J. G. Pereira, An Introduction to Geometrical Physics, World Scientific, Singapore, 1995.

12. A. G. Riess et al, Ap. J. 116, 1009 (1998); S. Perlmutter et al, Ap. J. 517, 565 (1999); P. de Bernardis et al, Nature 404, 955 (2000); S. Hanany et al, Ap. J. Letters 545, 5 (2000).

13. F. Gürsey, in Group Theoretical Concepts and Methods in Elementary Particle Physics, ed. by F. Gürsey, Istanbul Summer School of Theoretical Physics, Gordon and Breach, New York, 1962.

14. E. Inönü, in Group Theoretical Concepts and Methods in Elementary Particle Physics, ed. by F. Gürsey, Istanbul Summer School of Theoretical Physics, Gordon and Breach, New York, 1962. 
15. S. Coleman, Aspects of Symmetry, Cambridge University Press, Cambridge, 1985.

16. R. Aldrovandi and J. G. Pereira, A Second Poincaré Group, in Topics in Theoretical Physics: Festschrift for A. H. Zimerman, ed. by H. Aratyn et al, Fundação IFT, São Paulo, 1998 (Preprint gr-qc/9809061).

17. R. Aldrovandi, J. P. Beltrán Almeida and J. G. Pereira, Grav. Cosm. 11, 277 (2005) (Preprint gr-qc/0312017).

18. J. D. Jackson, Classical Electrodynamics, Wiley, New York, 1999.

19. S. Hossenfelder, Class. Quant. Grav. 231815 (2006) (Preprint hep-th/0510245).

20. G. Amelino-Camelia, Phys. Lett. B 510, 255 (2001) (Preprint hep-th/0012238); G. Amelino-Camelia, Int. J. Mod. Phys. D 11, 35 (2002) (Preprint gr-qc/0012051); J. Kowalski-Glikman, in Planck Scale Effects in Astrophysics and Cosmology, ed. by G. Amelino-Camelia and J. Kowalski-Glikman, Lect. Not. Phys. 669, 131 (2005) (Preprint hep-th/0405101).

21. V. A. Kostelecky (ed.), CPT and Lorentz, Symmetry, World Scientific, Singapore, 1999.

22. J. Kowalski-Glikman and S. Nowak, Class. Quant. Grav. 20, 4799 (2003) (Preprint hep-th/0304101).

23. F. Girelli and E. R. Livine, Some comments on the universal constant in DSR, Proceedings of DICE2006, Piombino, Italy (Preprint gr-qc/0612111).

24. F. Mansouri, Phys. Lett. B 538239 (2002) (Preprint hep-th/0203150).

25. G. Amelino-Camelia, Lect. Not. Phys. 541, 1 (2000) (Preprint gr-qc/9910089); R. J. Protheroe and H. Meyer, Phys. Lett. B 493, 1 (2000); S. Sarkar, Mod. Phys. Lett. A 17, 1025 (2002) (Preprint gr-qc/0204092); D. V. Ahluwalia, Mod. Phys. Lett. A 17, 1135 (2002) (Preprint gr-qc/0205121); T. Jacobson, S. Liberati and D. Mattingly, Phys. Rev. D 66, 081302 (2002) (Preprint hep-ph/0112207); R. C. Myers and M. Pospelov, Phys. Rev. Lett. 90, 211601 (2003) (Preprint hep-ph/0301124); R. H. Brandenberger and J. Martin, Int. J. Mod. Phys. A 17, 3663 (2002) (Preprint hep-th/0202142).

26. G. Amelino-Camelia, L. Smolin and A. Starodubtsev, Class. Quant. Grav. 21, 3095 (2004) (Preprint hep-th/0306134). 
Copyright of AIP Conference Proceedings is the property of American Institute of Physics and its content may not be copied or emailed to multiple sites or posted to a listserv without the copyright holder's express written permission. However, users may print, download, or email articles for individual use. 